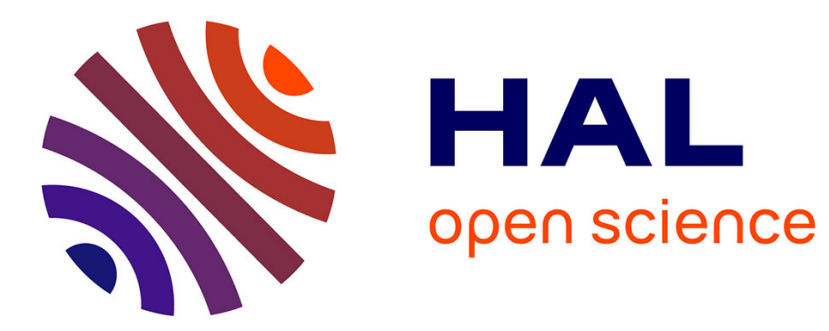

\title{
Influence of heterogeneities on crack propagation
}

Okan Yilmaz, Jeremy Bleyer, Jean-François Molinari

\section{To cite this version:}

Okan Yilmaz, Jeremy Bleyer, Jean-François Molinari. Influence of heterogeneities on crack propagation. International Journal of Fracture, 2018, 209 (1-2), pp.77-90. 10.1007/s10704-017-0239-6 . hal-01571293

\section{HAL Id: hal-01571293 \\ https://hal-enpc.archives-ouvertes.fr/hal-01571293}

Submitted on 2 Aug 2017

HAL is a multi-disciplinary open access archive for the deposit and dissemination of scientific research documents, whether they are published or not. The documents may come from teaching and research institutions in France or abroad, or from public or private research centers.
L'archive ouverte pluridisciplinaire HAL, est destinée au dépôt et à la diffusion de documents scientifiques de niveau recherche, publiés ou non, émanant des établissements d'enseignement et de recherche français ou étrangers, des laboratoires publics ou privés. 


\title{
Influence of heterogeneities on crack propagation
}

\author{
Okan Yllmaz $^{\mathrm{a}, *}$, Jérémy Bleyer ${ }^{\mathrm{a}}$, Jean-François Molinari ${ }^{\mathrm{a}}$ \\ ${ }^{a}$ Civil Engineering Institute, Materials Science and Engineering Institute, Ecole \\ Polytechnique Fédérale de Lausanne (EPFL), Station 18, CH-1015 Lausanne, Switzerland
}

\begin{abstract}
The influence of material heterogeneities is studied in the context of dynamic failure. We consider a pre-strained plate problem, the homogeneous case of which has been widely studied both experimentally and numerically. This setup is used to isolate the effects of the elastic field resulting from pre-straining and stress wave interactions throughout the crack propagation by adding stiffer and denser regions in the plate. While the crack tip is pushed away by stiffer inclusions, it is attracted to the denser ones. With the presence of denser media, only a portion of the total elastic energy in the system is effectively used to drive crack propagation, leading to a drop in the velocity of its tip in comparison to the homogeneous case. The crack velocity is shown to be an invalid criterion for crack branching. Instead, we introduce an effective stored energy to analyze the crack velocity and the emergence of crack branching instabilities.
\end{abstract}

Keywords: pre-strained plate, dynamic fracture, cohesive elements, crack propagation, branching

\section{Introduction}

Fracture prevention of materials and structures plays a key role in modern engineering designs. Understanding the physical mechanisms governing the dynamies of fracture in brittle and quasi-brittle media is thus erueial to improve existing procedures and design better products. However, this is still

\footnotetext{
* Corresponding author

Email address: okan.yilmaz@epfl.ch (Okan Yllmaz)
} 
a challenge due to the presence of material heterogeneities at different length scales, complexity of fracture process zone, and dynamic stress redistribution caused by the emitted stress waves from the moving crack tip and boundary reflections [1. Although elastodynamic linear fracture mechanics theory provides the time variation of the dynamic stress intensity factor as well as other field quantities, it is not adequate to capture the dynamic response of brittle materials 2. Experiments indicate several phenomena including limiting crack velocities below the Rayleigh wave speed of the material and crack branching at micro and macroscale that the linear theory fails to describe.

Especially for the evolution of crack dynamics, much of the theoretical and experimental work is done in homogeneous settings. Heterogeneity is present in materials at different scales, which are likely to introduce modifications to the homogeneous medium solutions [3, 4]. Material inhomogeneities have been studied at all scales from kilometers [5, 6] to nanometers [7, 8, the applications These various alterations to the dynamic process of fracturing are governed by the changes in the elastic field and stress wave emissions and reflections in the medium. Here, we focus on the evolution and propagation of fractures and the resulting crack patterns.

25 The objective of this paper is to examine the influence of distant heterogeneities on the dynamics of fracture. For this purpose, we numerically analyze the well-studied pre-strained plate configuration [9, 10, 11, 12, 13, 11. Stiffer and denser regions are introduced within this rapid fracture scheme to study their effect on the crack propagation velocity and crack branching instabilities.

In Section 2, the cohesive-element methodology, which is used to model fracture is explained. Section 3 presents a pre-strained rectangular plate problem using a homogeneous medium and the influence of the heterogeneities are studied in Section 4 Stiffer inclusions are added to alter the elastic field in Section 4.1. The strip is narrowed with denser regions in Section 4.2 to investi-

35 gate the influence of wave interactions while preserving the homogeneous elastic properties. Wave interactions are further studied with circular dense inclusions 
in Section 4.3 allowing comparisons with the cases having stiffer inclusions. Conclusions are given in Section 5.

\section{Fracture model}

Dynamic fracture is modeled using the cohesive-element methodology. The method is based on the cohesive crack model of Dugdale [14] and Barenblatt [15]. To represent the decohesion, we bury all complex debonding processes in a phenomenological, simple, cohesive law relating the traction and opening displacement. This constitutive response is called traction-separation law:

$$
\mathbf{T}=\mathbf{T}(\Delta)
$$

where $\mathbf{T}$ is the traction acting on the separating surfaces and $\Delta$ is the relative opening displacement vector. We use a simple linear irreversible softening law [16, 17. The existing free potential energy $\phi$, is assumed to depend only on one effective scalar displacement $\delta$ of the following form:

$$
\delta=\sqrt{\Delta_{n}^{2}+\beta^{2} \Delta_{s}^{2}},
$$

where $\Delta_{n}$ and $\Delta_{s}$ are the normal and tangential displacement components while $\beta$ is the parameter that couples these two displacements. This parameter can be estimated by imposing lateral confinement on specimens subjected to high strain rate axial compression [18 and roughly defines the ratio of the critical stress intensity factors of Mode II to Mode I of the material [19. The derivation of free potential energy with respect to the opening displacement gives the cohesive tractions:

$$
\mathbf{T}=\frac{\partial \phi}{\partial \boldsymbol{\Delta}}=\frac{T}{\delta}\left(\Delta_{n} \mathbf{n}+\beta^{2} \Delta_{s} \mathbf{s}\right),
$$

where $\mathbf{n}$ and $\mathbf{s}$ are the unit vectors in normal and tangential directions. Crack opening condition is denoted with $\delta=\delta_{\max }$ and $\dot{\delta}>0$, and crack closure and reopening case is stated as $\delta<\delta_{\max }$. Thus, the traction is

$$
T=\left\{\begin{array}{ll}
f_{\mathrm{ct}}\left(1-\frac{\delta}{\delta_{\mathrm{c}}}\right) & \text { for } \delta=\delta_{\max } \text { and } \dot{\delta}>0 \\
\frac{\delta}{\delta_{\max }} T_{\max } & \text { for } \delta<\delta_{\max }
\end{array},\right.
$$


where $f_{\mathrm{ct}}$ is the critical stress associated to the cohesive element, $\delta_{\mathrm{c}}$ is the effective relative displacement value beyond which complete decohesion occurs, $\delta_{\max }$ is the maximum value of the achieved effective displacement up to that instant and $T_{\max }$ is the associated traction value for maximum relative displacement.

The cohesive law and the areas that are associated to the energy dissipation are illustrated in Figure 1. Cohesive elements are dynamically inserted at the inter-element boundaries when and where the local stress exceeds a critical stress threshold, which is the tensile strength of the corresponding material. The irreversible part of dissipated energy is shown with the dark grey area and denoted by $E_{\text {irrev }}$ while the reversible part is the light grey area named $E_{\mathrm{rev}}$. Damaging of the cohesive element is an irreversible process while tractionseperation law allows recovering energy upon unloading. Damage in a cohesive element is denoted as:

$$
D=\min \left(\frac{\delta_{\max }}{\delta_{\mathrm{c}}}, 1\right)
$$

A simple penalty model is incorporated in the cohesive law for the case of compression:

$$
T_{n}=\alpha_{p} \Delta_{n} \quad \text { if } \quad \Delta_{n}<0,
$$

where $\alpha_{p}$ is the stiffness parameter and $T_{n}$ is the normal component of the 45 traction vector.

The total dissipated fracture energy during the separation process is given by the entire grey region. When the dissipated energy is equal to the total area under the curve shown in the figure, which is $\frac{1}{2} f_{\mathrm{ct}} \delta_{\mathrm{c}}$, full decohesion occurs and two free surfaces are formed at the interelement boundaries.

\section{Pre-strained rectangular plate}

The pre-strained rectangular plate problem was studied experimentally by 9 ] using PMMA and by [11, 20, 21, 12] using polyacrylamide gels. Several numerical investigations can be found using different fracture modeling approaches such as cohesive elements [10, non-local damage modeling [13], and phase-field 55 modeling [1]. 


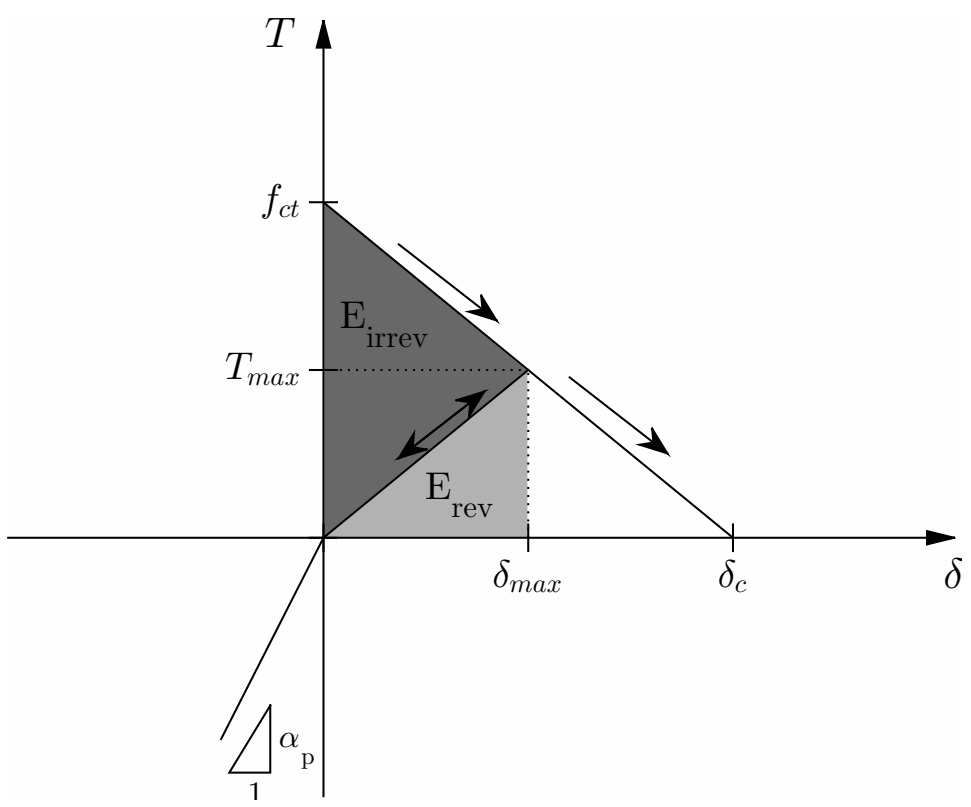

Figure 1: Graphical representation of linear irreversible cohesive law [16, 17]. Arrows denote loading and unloading directions.

The model experiment is conducted as follows. First, the plate is pre-strained by moving the upper and lower boundaries to the desired displacement. In this strained state, a sharp crack is created in the middle of one side of the sample using a razor edge. Pre-notched version of the identical setup is also popular and 60 widely studied both experimentally $[22,23,24,25]$ and numerically $[26,27,28]$. The only difference with the pre-strained condition is that the crack is seeded in the specimen before applying the load. For the plate geometry, pre-straining conditions, and crack size, see Figure 2

PMMA is used as the plate material, see Table 1 for the material parameters. The coupling parameter $\beta$ for the cohesive-element method is chosen to be 1 for this uniaxial tension case. Using the finite-element method, we solve the equation of motion

$$
\mathbf{M u ̈}+\mathbf{f}^{\text {int }}=\mathbf{f}^{\text {ext }},
$$

where $\mathbf{M}$ is the mass matrix, $\mathbf{u}$ is the displacement vector, and $\mathbf{f}^{\text {int }}$ and $\mathbf{f}^{\text {ext }}$ are the internal and external force vectors respectively. This equation is solved at 


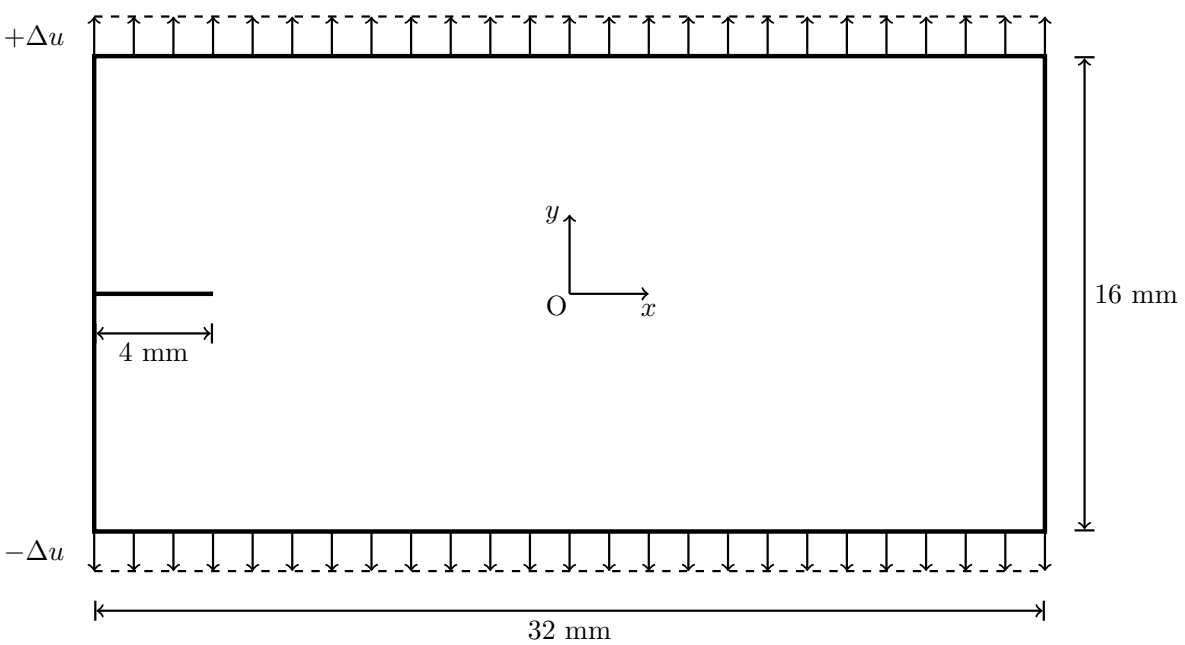

Figure 2: Pre-strained PMMA plate problem with an applied displacement $\Delta u$ to the top and bottom boundaries.

every time step using an explicit time integration scheme and the displacement field is updated. For further details regarding the implementation see [29]. The finite-element code used for the study is based on a Newmark explicit time integration scheme, which is conditionally stable when the time step is less than a critical value set by the Courant-Friedrichs-Lewy condition [30]:

$$
\Delta t_{\mathrm{crit}}=\alpha \min _{e=1 \ldots N}\left(\frac{l_{e}}{c}\right)
$$

where $c$ is the pressure wave speed of the associated material, $l_{e}$ is the size of element $e$, and $N$ is the total number of elements. The smallest value of the calculated time step over all elements is the chosen time step for the simulation. A security coefficient, $\alpha$, is also used ( 0.2 for this study) to ensure stability. A two-dimensional model with plane-stress assumption is used. Rayleigh wave speed of the material is calculated using the following formula:

$$
c_{R}=\frac{0.862+1.14 \nu}{1+\nu} c_{s}
$$

where $c_{s}$ is the shear wave speed, $c_{s}=\sqrt{\mu / \rho}$. For PMMA, $c_{s}=984.8 \mathrm{~m} / \mathrm{s}$ and ${ }_{65} \quad c_{R}=919.9 \mathrm{~m} / \mathrm{s}$. 
Cohesive-zone modeling introduces another length scale set by the elastic and fracture properties. This length scale can be expressed for simple cases (mode I loading, homogeneous material) as [31, 32]:

$$
l_{z}=\frac{E G_{\mathrm{c}}}{f_{\mathrm{ct}}^{2}} .
$$

The condition $l_{\mathrm{m}}<l_{\mathrm{z}}$ between the length scales has to be satisfied in our simulations where $l_{\mathrm{m}}$ is the biggest element size. The cohesive zone must contain several elements (typically around four [33]) and should be small compared to the sample size for mesh independency. The cohesive zone length for PMMA material is calculated as $0.23 \mathrm{~mm}$. A mesh of average mesh size $l_{\mathrm{av}}=0.05 \mathrm{~mm}$ is used to correctly resolve the cohesive zone in front of the crack tip.

Table 1: Material properties of PMMA 10, 1.

\begin{tabular}{lc}
\hline & PMMA \\
\hline \hline Young's modulus, $E(\mathrm{GPa})$ & 3.09 \\
Poisson's ratio, $\nu$ & 0.35 \\
Density, $\rho\left(\mathrm{kg} / \mathrm{m}^{3}\right)$ & 1180 \\
\hline Fracture energy, $G_{\mathrm{c}}\left(\mathrm{J} / \mathrm{m}^{2}\right)$ & 300 \\
Tensile strength, $f_{\mathrm{ct}}(\mathrm{MPa})$ & 75 \\
Coupling parameter, $\beta$ & 1 \\
\hline
\end{tabular}

Figure 3 illustrates the crack patterns at displacements from $\Delta u=0.035 \mathrm{~mm}$ to $\Delta u=0.10 \mathrm{~mm}$. At lower displacements, there exists only a single crack propagating from the initial cut. However, increasing the level of pre-straining, we observe first microbranching and an increase in the damage band. Microbranches are defined as the local branching events where main crack sprouts side branches that propagate for a short distance and die [24]. This is a numerical instability observed beyond a critical velocity, which [34] observed around $0.4 c_{R}$. The limiting velocity is in fact a result of this instability as it prevents the crack to further accelerate while energy dissipation is not limited to a single propagating crack tip [1. In addition, local branching events turn into mac- 
robranches, which do not die right away and can propagate in the medium for some time when $\Delta u \geq 0.06 \mathrm{~mm}$.

The cohesive-element approach is known to exhibit a mesh dependency on the dissipated energy, thus the amount of microcracking, especially for coarse meshes [35. In this study, we use meshes that are fine enough to fully resolve the cohesive zone in front of the crack tip with the help of parallel computing. However, a convergence analysis of the dissipated energy with the mesh size is missing. Nonetheless, salient experimental features of the dynamic crack propagation scheme such as the limiting crack velocity and branching at higher pre-straining levels [9, 10 may be captured using cohesive elements.

The snapshots in Figure 3 are taken when the crack fully propagates from one end to the other. The crack needs less time to reach the right end of the plate at higher pre-straining levels. Crack propagation velocities as a function of $x$-position of the crack tip are plotted in Figure 4. The instantaneous crack velocity is calculated by fitting a linear function for several consecutive time steps versus crack tip position around that particular location.

The velocities are higher than the experimentally observed limiting velocities. It was shown by [10] that the rate-independent cohesive law wrongly predicts Rayleigh wave speed of the material, $c_{R}$ as the limiting velocity, which is reproduced by our model. However, experiments show that the limiting velocities are in the range of $0.5-0.65 c_{R}$ for glass, $0.58-0.62 c_{R}$ for PMMA, and $0.3-0.45 v / c_{R}$ for Homalite-100, see [2] and references therein. This velocity also depends on the experimental setup for a given material. Zhou et al. [10] also showed that the experimentally observed limiting velocities can be obtained with a phenomenological rate-dependent cohesive law. Since this study focuses on the effects of heterogeneities, the rate-independent scheme is preserved.

Crack propagation in this setting can be altered by two mechanisms. First is the changes in the elastic field, which can be obtained by introducing inclusions of different elastic properties. Wave dynamics can also influence crack propagation. To have different stress wave interaction in an identical elastic field, regions with same elastic modulus but different mass density will be introduced. 


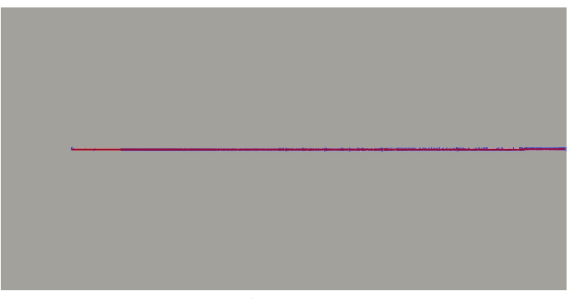

damage

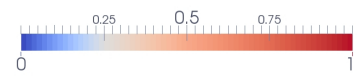

(a)

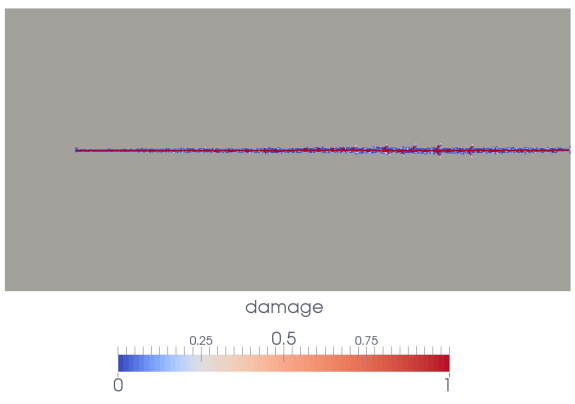

(c)

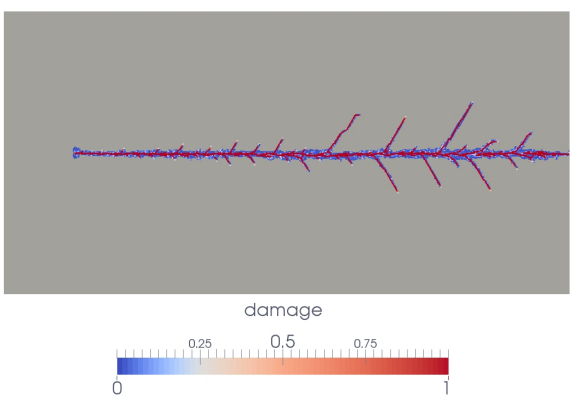

(e)

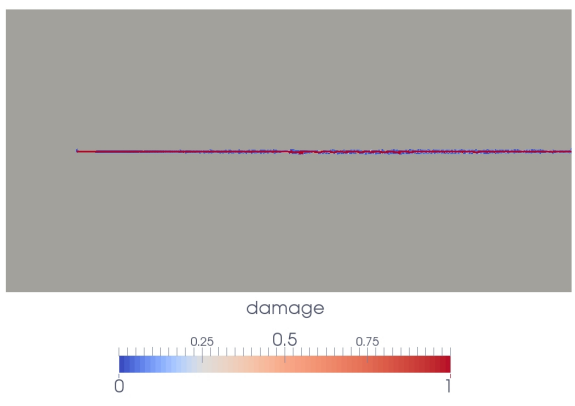

(b)

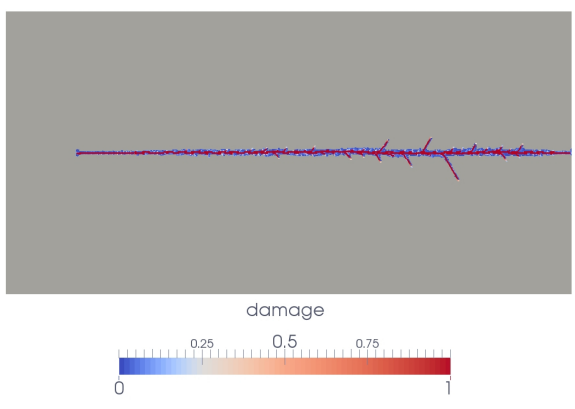

(d)

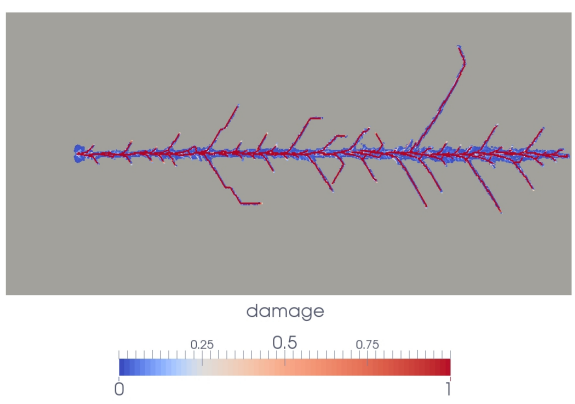

(f)

Figure 3: Crack propagation with different pre-strain loadings. The snapshots are taken at times (a) $t=4.53 \times 10^{-5} \mathrm{~s}$ (b) $t=4.30 \times 10^{-5} \mathrm{~s}(\mathrm{c}) t=4.07 \times 10^{-5} \mathrm{~s}(\mathrm{~d}) t=3.85 \times 10^{-5} \mathrm{~s}(\mathrm{e})$ $t=3.62 \times 10^{-5} \mathrm{~s}(\mathrm{f}) t=3.40 \times 10^{-5} \mathrm{~s}$. The applied displacements are (a) $\Delta u=0.035 \mathrm{~mm}$ (b) $\Delta u=0.04 \mathrm{~mm}$ (c) $\Delta u=0.05 \mathrm{~mm}$ (d) $\Delta u=0.06 \mathrm{~mm}$ (e) $\Delta u=0.08 \mathrm{~mm}$ (f) $\Delta u=0.10 \mathrm{~mm}$. 


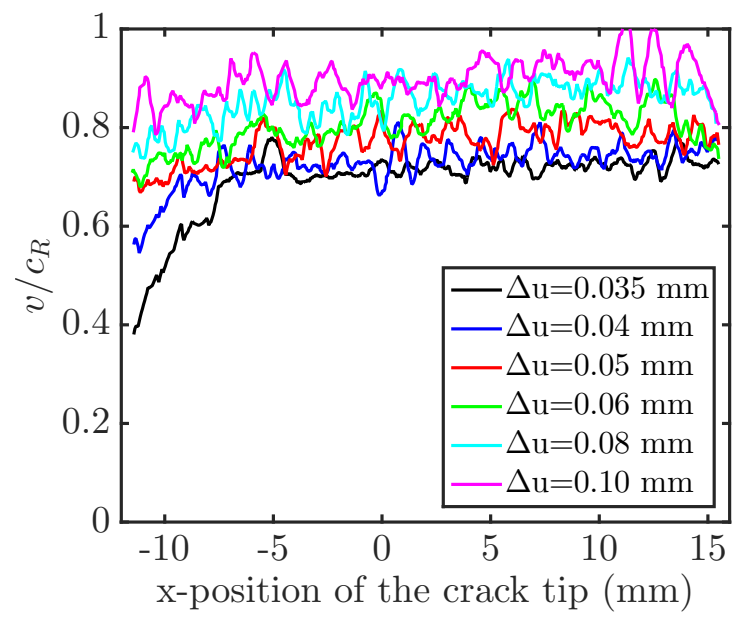

Figure 4: Crack velocity normalized with the Rayleigh wave speed of the material as a function of crack tip position for different levels of pre-straining the homogeneous PMMA plate given in Figure 2

\section{Influence of heterogeneities}

\subsection{Stiff inclusions}

The pre-strained elastic field is changed by adding a series of circular inclusions, see Figure 5. Elastic modulus of inclusions is ten times the plate material, $E_{\text {inc }}=10 E_{0}=30.9 \mathrm{GPa}$. Boundary conditions are identical to the homogeneous case. $l_{x}$ is the $x$-distance from the initial crack tip to the centerline of the first inclusion, $l_{y}$ is the $y$-distance from the plate centerline to the bottom tangent of the inclusions and $l_{c}$ is the distance between centers of the inclusions. The first inclusion is $l_{x}=3 \mathrm{~mm}$ away from the initial crack tip and the distance between the inclusions is $l_{c}=3 \mathrm{~mm}$. These two dimensions are kept constant while the inclusion size and distance to the plate centerline are varied.

Figure 6 shows crack propagation for different inclusion size and centerline proximity. The displacement is $\Delta u=0.035 \mathrm{~mm}$ is applied for all cases to have a single propagating crack.

We observe that the crack is pushed away by the stiff inclusions. This deflection increases when the diameters of the inclusions are larger. Total deflections 


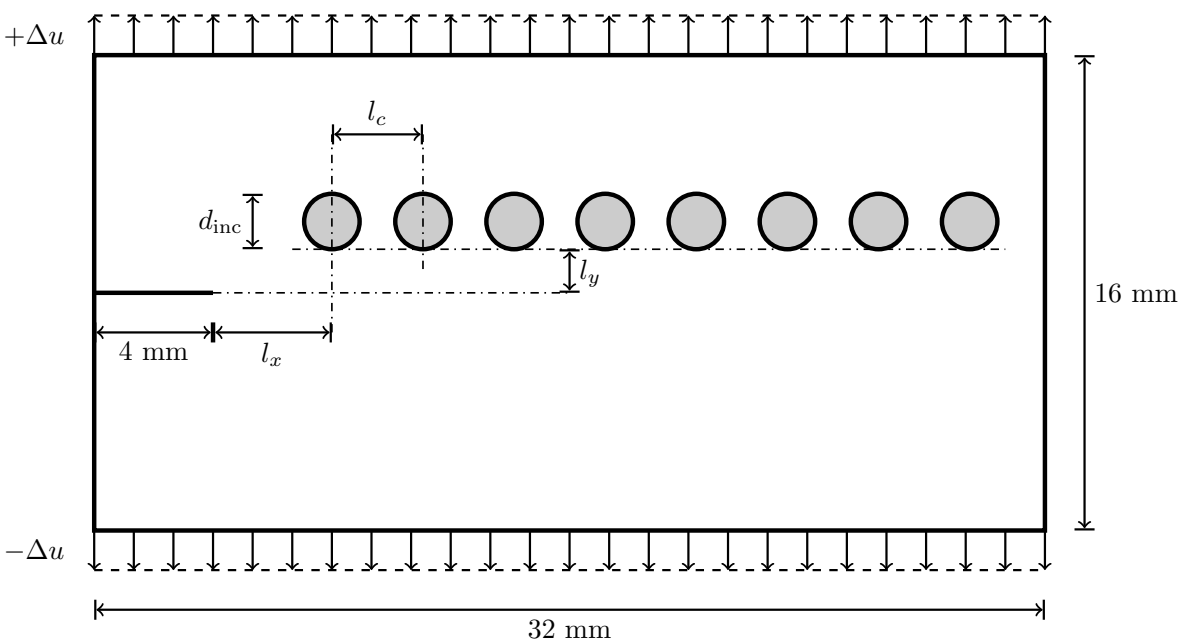

Figure 5: Pre-strained PMMA plate problem with the addition of stiff inclusions of Young's modulus $E_{\mathrm{inc}}=10 E_{0}$ and diamater $d_{\mathrm{inc}}$. The density of the inclusions is identical to PMMA. $l_{x}$ is the $x$-distance from the initial crack tip to the centerline of the first inclusion, $l_{y}$ is the $y$-distance from the plate centerline to the bottom tangent of the inclusions and $l_{c}$ is the distance between centers of the inclusions. The applied displacement is $\Delta u$ and boundary conditions are identical to Figure 2

from the centerline are $0.4 \mathrm{~mm}$ for $d_{\mathrm{inc}}=1 \mathrm{~mm}$ and $0.7 \mathrm{~mm}$ for $d_{\mathrm{inc}}=2 \mathrm{~mm}$. Pushing the inclusions closer to the centerline ( $l_{y}$ from 0.5 to 0.1 ) does not have a prominent influence to the total deflection, they are measured in this case $0.5 \mathrm{~mm}$ and $0.7 \mathrm{~mm}$ respectively. No significant change in crack velocity is observed with the stiff inclusions.

\subsection{Dense strip}

Crack propagation may also be influenced by the elastic wave interactions during its movement. The pre-strained plate is a suitable setup to control stress wave dynamics as the boundaries are fixed at a given displacement and only the propagating crack tip emits stress waves. Here, we introduce regions ten times denser than PMMA material, $\rho_{d}=10 \rho_{0}$ in the upper and lower part of plate keeping the elastic modulus constant. Thus, the initial elastic field is identical to the homogeneous case and only wave interactions can modify the crack. The 


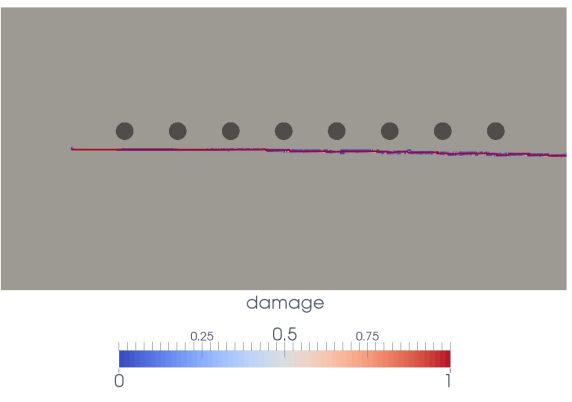

(a)

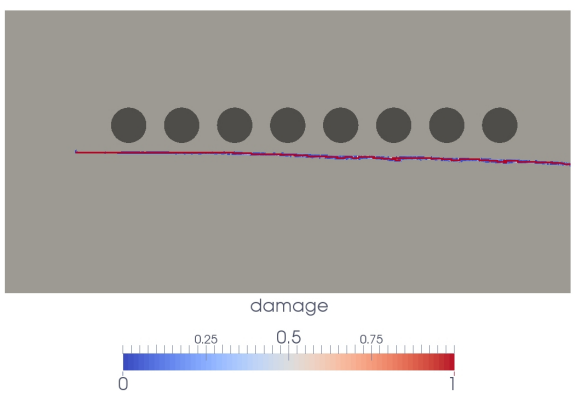

(c)

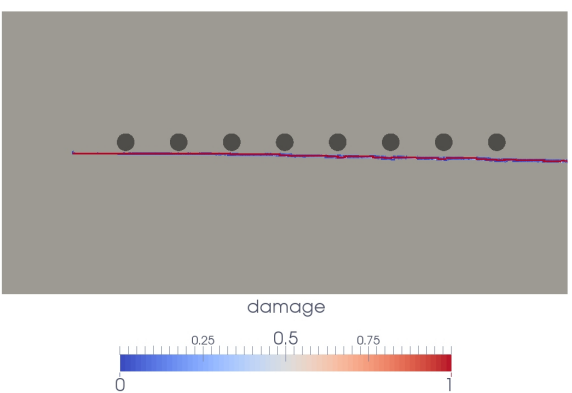

(b)

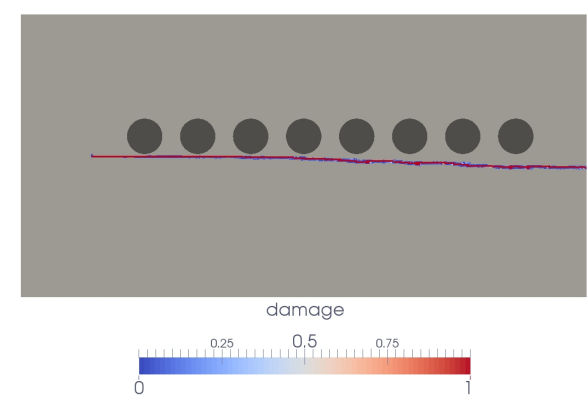

(d)

Figure 6: Crack propagation in different inclusion pattern configurations with the inclusions having the same density but different Young's modulus $E_{\text {inc }}=10 E_{0}$. The applied displacement is $\Delta u=0.035 \mathrm{~mm}$ for all cases. The snapshots are taken at times (a) $t=4.67 \times 10^{-5} \mathrm{~s}$ (b) $t=4.67 \times 10^{-5} \mathrm{~s}$ (c) $t=4.55 \times 10^{-5} \mathrm{~s}(\mathrm{~d}) t=4.55 \times 10^{-5} \mathrm{~s}$. The diameters and distance from the centerline are (a) $d_{\text {inc }}=1 \mathrm{~mm}, l_{y}=0.5 \mathrm{~mm}$ (b) $d_{\text {inc }}=1 \mathrm{~mm}, l_{y}=0.1 \mathrm{~mm}$ (c) $d_{\text {inc }}=2 \mathrm{~mm}, l_{y}=0.5 \mathrm{~mm}(\mathrm{~d}) d_{\text {inc }}=2 \mathrm{~mm}, l_{y}=0.1 \mathrm{~mm}$. The first inclusion is $l_{x}=3 \mathrm{~mm}$ away from the initial crack tip and the distance between the inclusions is $l_{c}=3 \mathrm{~mm}$.

model is sketched in Figure 7. Again, boundary conditions are identical to the homogeneous case.

Dense media change wave dynamics considerably. The crack tip emits compressive waves as it propagates. Without denser regions, these waves hit the fixed boundaries and reflect fully as tensile waves. However, hitting a boundary with a different density, some portion of the wave is transmitted to the following medium and the remaining part is reflected back. Using the impedance ratio $R$, we can estimate the amount of transmitted and reflected wave. This ratio is 


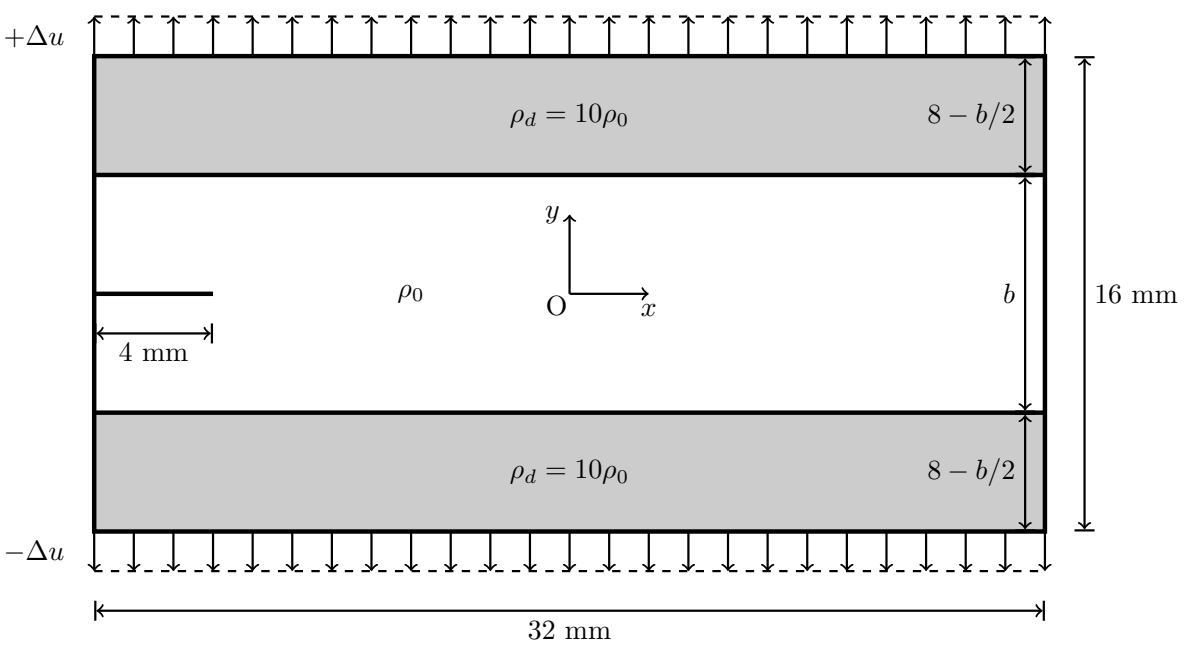

Figure 7: Pre-strained PMMA plate problem modified with dense regions. The density of grey regions is ten times the density of PMMA, $\rho_{d}=10 \rho_{0}$. The stiffness is identical for both regions, $E_{d}=E_{0}$. The applied displacement is $\Delta u$ and boundary conditions are identical to Figure 2

calculated for the given material values as

$$
R=\frac{c_{d} \rho_{d}}{c_{0} \rho_{0}}=\frac{\rho_{d}}{\rho_{0}} \sqrt{\frac{E_{d} \rho_{0}}{\rho_{d} E_{0}}}=\sqrt{\frac{\rho_{d}}{\rho_{0}}}=\sqrt{10} .
$$

Assuming one-dimensional stress-wave propagation of magnitude $f_{I}$, reflected and transmitted waves are found using the impedance ratio:

$$
f_{R}=f_{I} \frac{R-1}{R+1}=0.52 f_{I}, \quad f_{T}=f_{I} \frac{2 R}{R+1}=1.52 f_{I}
$$

At the boundary of dense region, a compressive wave is reflected back as a compressive wave of half the magnitude and transmitted to the dense region with a higher magnitude. Transmitted compressive wave is then reflected back from the plate boundary as a tensile wave, however travels at a lower speed since the medium is denser. Entering back to the PMMA region, the impedance ratio is reversed. With $R=1 / \sqrt{10}$, the reflection coefficient is -0.52 and transmission coefficient is 0.48 . Hence, approximately $73 \%$ of the compressive wave emitted returns back as a tensile wave when it travels to the simulation-box boundary and back. Note that this is valid for only one wave in a one-dimensional setting, 
thus with continuous wave emission from a fast moving crack tip, the wave interaction picture is much more complex.

Figure 8 shows crack velocities for various PMMA widths (b parameter). The plots are grouped under the levels of pre-straining. In all cases we observe that crack velocity decreases as $b$ gets narrower. Apart from this global decrease, local drops are observed.
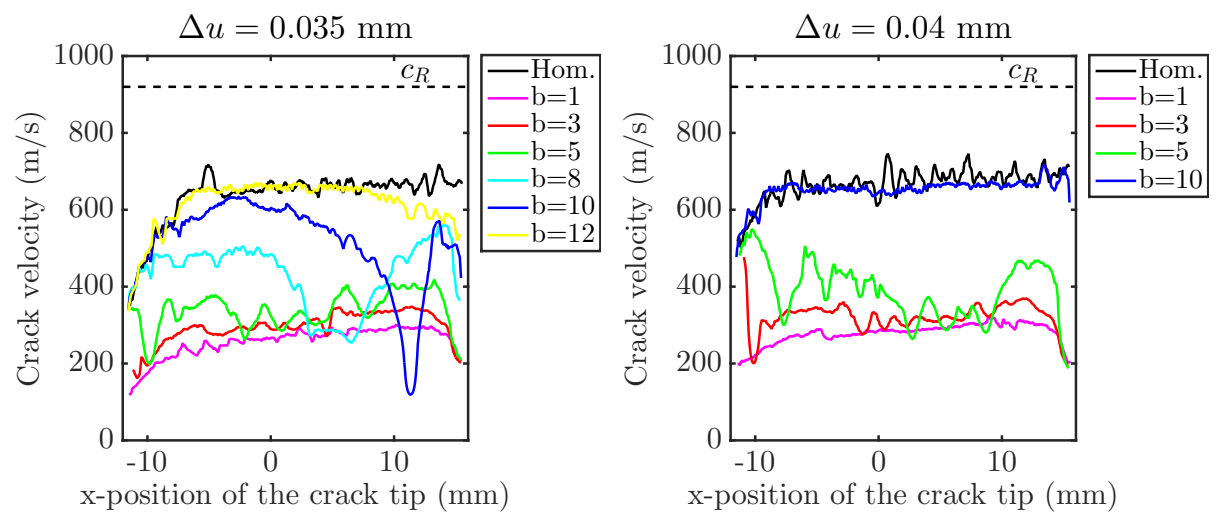

(a)

(b)
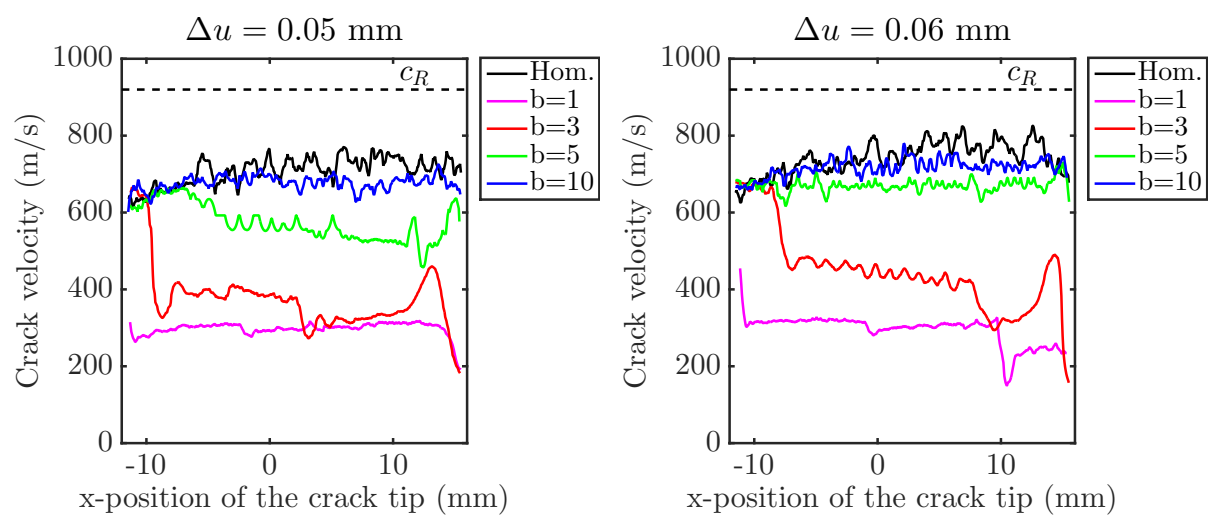

(c)

(d)

Figure 8: Crack velocities as a function of crack tip position for different dense region configurations, $b$-widths $(\mathrm{mm})$ in the legends. The applied displacements are (a) $\Delta u=0.035 \mathrm{~mm}$ (b) $\Delta u=0.04 \mathrm{~mm}$ (c) $\Delta u=0.05 \mathrm{~mm}$ (d) $\Delta u=0.06 \mathrm{~mm}$. The horizontal dashed line is the Rayleigh wave speed of the material. In all cases it is observed that decreasing $b$, the crack velocity decreases. Apart from this global decrease, local drops are also observed, see cases $b=8$ and $10 \mathrm{~mm}$ in (a). 
This setup is closer to the infinite strip problem [36, 37] than the infinite be a meaningful criterion.

The initially stored energy is a well-defined property of the system and can be related to dynamic parameters such as the crack tip velocity or fracture energy 


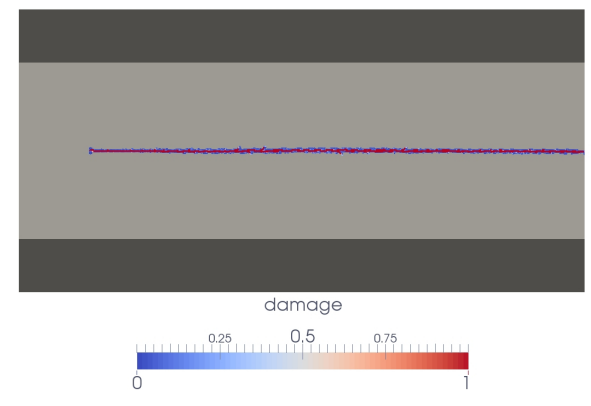

(a)

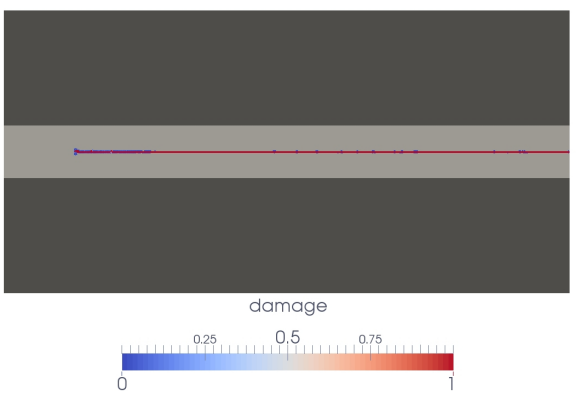

(c)

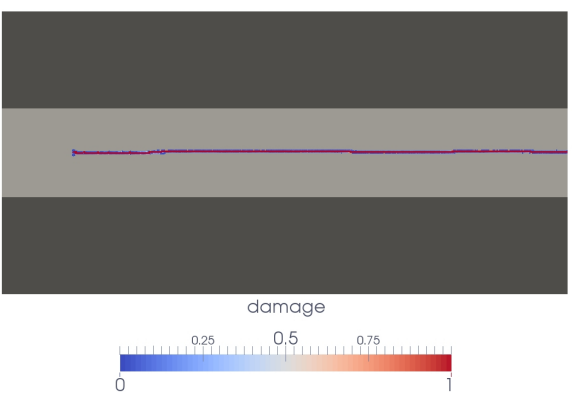

(b)

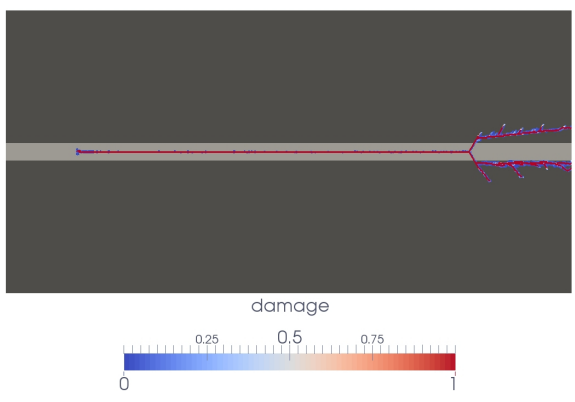

(d)

Figure 9: Crack propagation in different dense plate configurations. Grey areas denote the PMMA material and black areas are for the denser region with $\rho_{d}=10 \rho_{0}$. The applied displacement is $\Delta u=0.06 \mathrm{~mm}$ for all cases. The snapshots are taken at times (a) $t=$ $4.01 \times 10^{-5} \mathrm{~s}$ (b) $t=4.32 \times 10^{-5} \mathrm{~s}$ (c) $t=6.91 \times 10^{-5} \mathrm{~s}(\mathrm{~d}) t=1.03 \times 10^{-4} \mathrm{~s}$. The width of the confined PMMA regions are (a) $b=10 \mathrm{~mm} \mathrm{(b)} b=5 \mathrm{~mm} \mathrm{(c)} b=3 \mathrm{~mm}$ (d) $b=1 \mathrm{~mm}$.

dissipated by crack advance [1. For the pre-strained plate configuration, total stored energy per specimen width is calculated as

$$
W=\frac{2 E(\Delta u)^{2}}{h}
$$

Figure 10a shows the crack velocities versus the total stored energy. Filled points indicate the homogeneous plate cases while hollow points are the cases with $b<16 \mathrm{~mm}$. Although the set of points corresponding to a particular pre-straining level indicate different crack velocities, total stored energy is identical. Introducing dense regions do not change the total energy available in the system as it is only a function of stiffness (constant in all cases) and applied 
displacement.

One can attain much lower velocities when the propagating crack is surrounded with denser regions. and this is proportional to their amount. In other words, These regions limit the elastic energy, which can be used to propagate the crack tip. To explain the difference in the failure dynamics, we introduce an effective stored energy, which is the multiplication of total stored energy by $b / h$, the ratio of actual PMMA volume in the plateto the denser material:

$$
W_{\text {eff }}=\frac{2 E(\Delta u)^{2}}{h} \frac{b}{h} \text {. }
$$

Using the effective stored energy instead of the total one, the points in Figure 10a fall remarkably on the same curve, see Figure 10p. Thus, the given density ratio is able to trap the corresponding portion of the mechanical potential energy of the system to the denser regions where mechanical waves travel slower, therefore limiting the amount to be used for crack propagation.

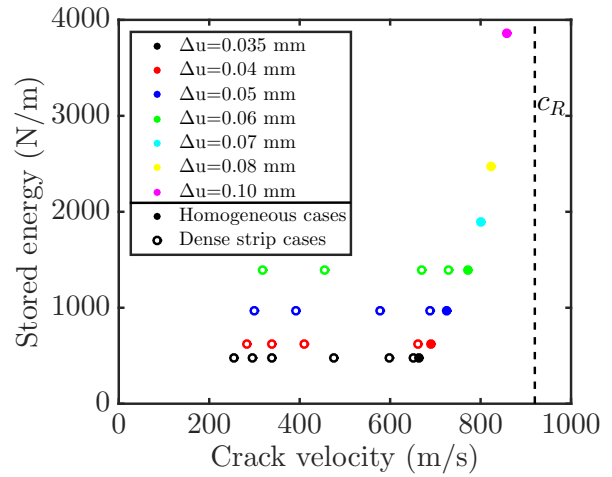

(a)

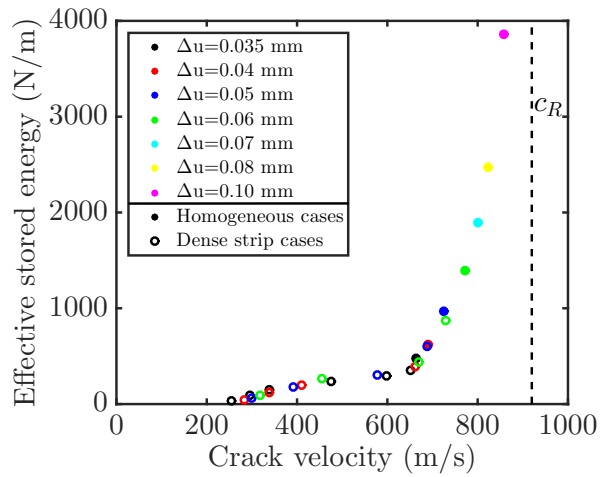

(b)

Figure 10: Stored energy per unit length $W$ (a) as a function of crack velocity. Legend shows the applied displacement. Filled circles represent the homogeneous cases while hollow circles are the cases where $b<16 \mathrm{~mm}$. Using the effective stored energy per unit length $W_{\text {eff }}$ (b) we observe that the points fall on the same curve. The vertical dashed line is the Rayleigh wave speed of the material.

Dividing the total dissipated energy by the distance from the initial crack tip to the end of the plate $(28 \mathrm{~mm})$, we get the specific fracture energy, which is plotted in Figure 11 for different $b$ and $\Delta u$ with respect to the crack velocity 
and effective stored energy. The outlier point is the case $\Delta u=0.06 \mathrm{~mm}$, $b=1 \mathrm{~mm}$, at which crack branching is observed at a low velocity, see Figure $9 \mathrm{~d}$ for the pattern. The material property $G_{c}$ constitutes a lower bound for the normalized dissipated energy in the system. Above this limit, local branching events start, which is found to be around $650 \mathrm{~m} / \mathrm{s}$. With the increasing energy dissipation capability of the system, we observe macrobranches, which can also occur at low velocities.

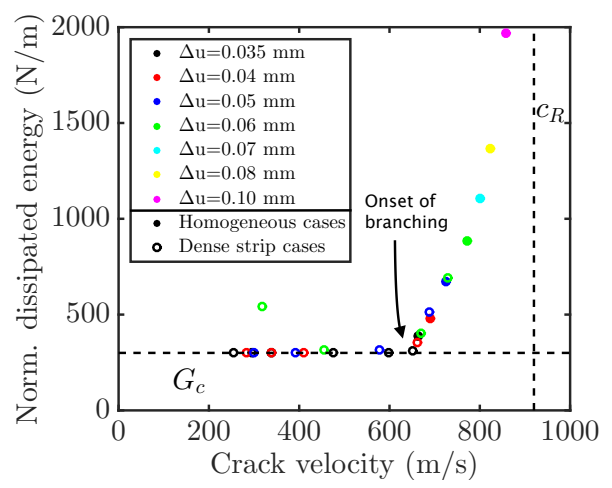

(a)

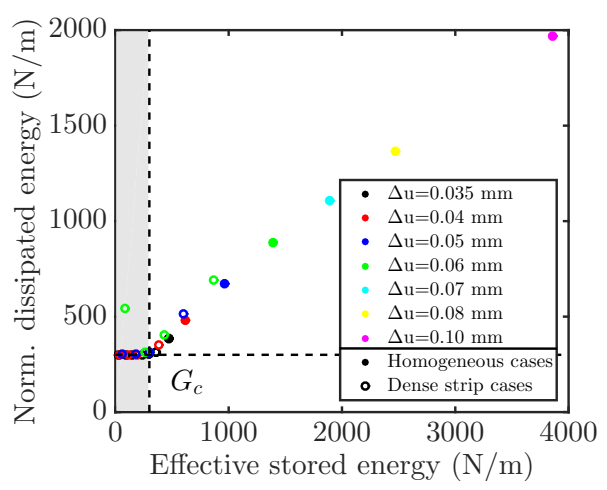

(b)

Figure 11: Total dissipated energy normalized with the distance from the initial crack tip to the plate end $(0.028 \mathrm{~m})$ versus the crack velocity (a) and effective stored energy (b). Filled circles represent the homogeneous cases while hollow circles are the cases where $b<16 \mathrm{~mm}$. (a) A lower bound of $300 \mathrm{~J} / \mathrm{m}^{2}$, which is the material property of the traction-separation law is shown with a horizontal dashed line. The vertical dashed line is the Rayleigh wave speed of the material. (b) Both the horizontal and vertical dashed lines denote the limit $G_{\mathrm{C}}=300 \mathrm{~J} / \mathrm{m}^{2}$. It is possible to have crack propagation in the grey area $\left(W_{\text {eff }}<G_{\mathrm{C}}\right.$ ) as the total stored energy is higher than the effective one.

Figure $11 \mathrm{p}$ shows the relation between the dissipated and effective stored energies. In this case, both the horizontal and vertical limits denote the material fracture energy, $G_{\mathrm{c}}=300 \mathrm{~J} / \mathrm{m}^{2}$. An explanation for the initiation of the local branching events is provided by the effective stored energy. We observe that when $W_{\text {eff }}>G_{\mathrm{c}}$, microbranching starts. It is possible to have crack propagation when $W_{\text {eff }}<G_{\mathrm{C}}$ (the grey-colored area), since the total available elastic energy in the plate is higher as shown before in Figure $10 \mathrm{a}$. 


\subsection{Dense inclusions}

Lastly, we consider circular shaped dense inclusions for direct comparison with stiff inclusions. The scheme in Figure 5 is used but with identical elastic modulus and density of inclusions $\rho_{\mathrm{inc}}=10 \rho_{0}$. Only $l_{y}=0.1 \mathrm{~mm}$ case is compared. The applied displacement is $\Delta u=0.035 \mathrm{~mm}$ to prevent any branching.

Figure 12 illustrates the crack propagation for two different inclusion sizes. Unlike the original stiffer inclusions case (see Section 4.1), the crack tip is attracted to the denser inclusions and this attraction is proportional to the inclusion size.
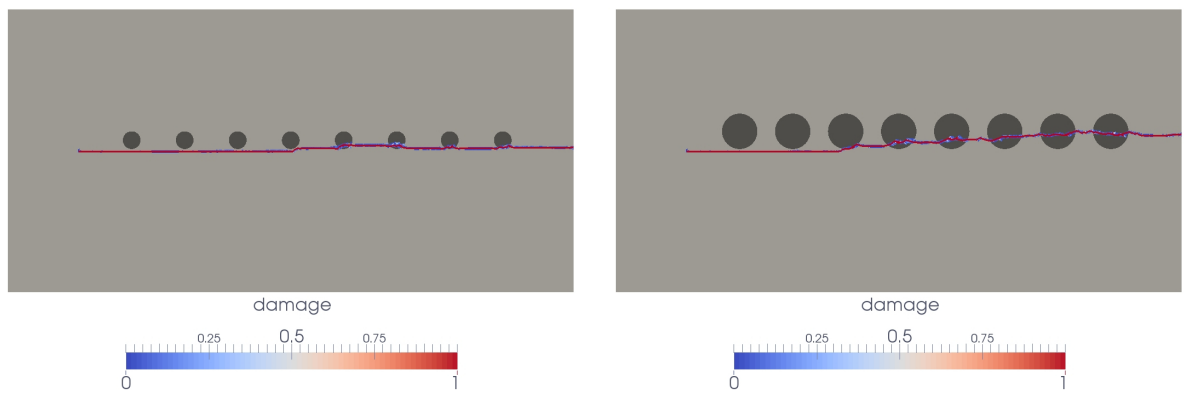

(a)

(b)

Figure 12: Crack propagation in different inclusion pattern configurations with the inclusions having the same Young's modulus but a density of $\rho_{\text {inc }}=10 \rho_{0}$. The snapshots are taken at times (a) $t=6.74 \times 10^{-5} \mathrm{~s}$ (b) $t=9.93 \times 10^{-5} \mathrm{~s}$. The diameters are (a) $d_{\text {inc }}=1 \mathrm{~mm}$ (b) $d_{\text {inc }}=2 \mathrm{~mm}$ and the distance to the centerline is $l_{y}=0.1 \mathrm{~mm}$ in both cases. The first inclusion is $l_{x}=3 \mathrm{~mm}$ away from the initial crack tip and the distance between the inclusions is $l_{c}=3 \mathrm{~mm}$.

The attraction of the crack tip can be explained by the stress wave interactions creating energy favored directions. Figure 13 shows the evolution of strain energy density.

We observe that the strain energy density is higher at the upper portion of the crack tip vicinity as it propagates. The crack tip can be located in each snapshot by tracking the localized region of strain energy density. The deflection in the energy-favorable direction is expected as the Young's modulus 


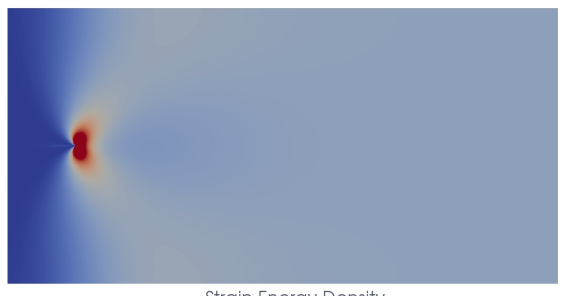

Strain Energy Density

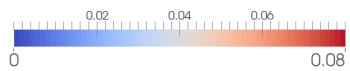

(a)

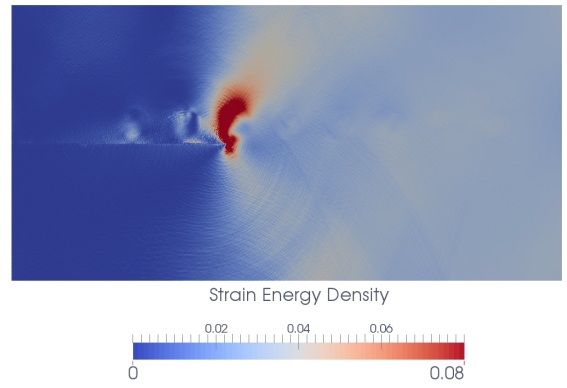

(c)

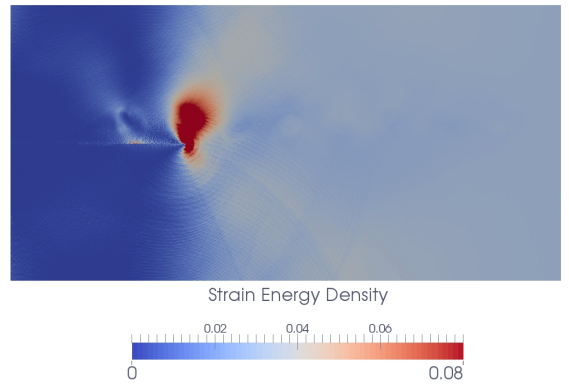

(b)

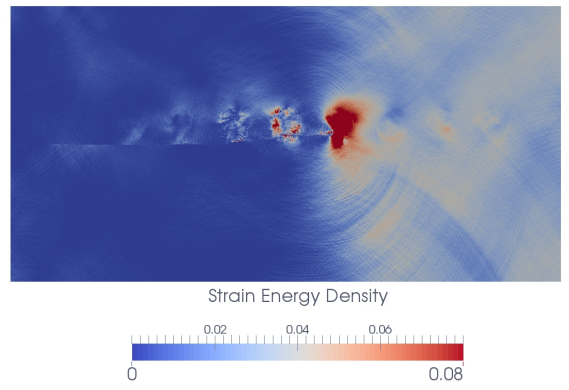

(d)

Figure 13: Strain energy density $\left(\mathrm{N} / \mathrm{mm}^{2}\right)$ at times (a) $t=0 \mathrm{~s}$ (b) $t=1.75 \times 10^{-5} \mathrm{~s}(\mathrm{c})$ $t=2.34 \times 10^{-5} \mathrm{~s}(\mathrm{~d}) t=4.68 \times 10^{-5} \mathrm{~s}$ with the inclusions having the same Young's modulus but a density of $\rho_{\text {inc }}=10 \rho_{0}$. The diameters are $d_{\text {inc }}=2 \mathrm{~mm}$ and the distance to the centerline is $l_{y}=0.1 \mathrm{~mm}$. The first inclusion is $l_{x}=3 \mathrm{~mm}$ away from the initial crack tip and the distance between the inclusions is $l_{c}=3 \mathrm{~mm}$ (the configuration shown in Figure $12 \mathrm{p}$ ).

is constant throughout the plate. The contribution of the kinetic energy is found to be insignificant compared to the strain energy.

The velocity of the crack tip is plotted in Figure 14. When the crack is passing by an inclusion it slows down and this effect is also proportional to the inclusion size. The crack tip is attracted to the dense inclusions and it passes through them during the analysis. The drop in the velocity is considerably higher while the crack propagates in the denser inclusion as the material wave speed is $\sqrt{\rho_{\text {inc }} / \rho_{0}}$ times lower. 


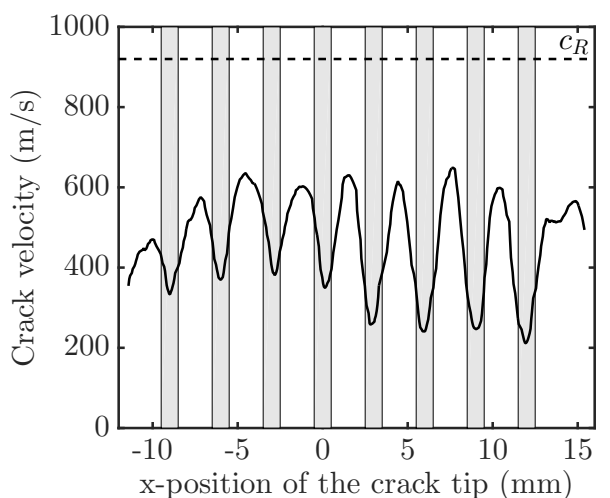

(a)

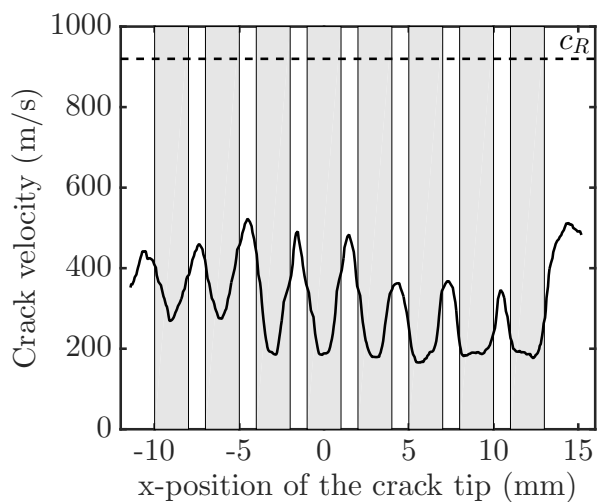

(b)

Figure 14: Crack velocity as a function of crack tip position for different inclusion pattern configurations with the inclusions having the same Young's modulus but a density of $\rho_{\text {inc }}=$ $10 \rho_{0}$. Inclusion positions are colored with grey. The diameters are (a) $d_{\text {inc }}=1 \mathrm{~mm}$ (b) $d_{\text {inc }}=2 \mathrm{~mm}$ and the distance to the centerline is $l_{y}=0.1 \mathrm{~mm}$ in both cases. The first inclusion is $l_{x}=3 \mathrm{~mm}$ away from the initial crack tip and the distance between the inclusions

is $l_{c}=3 \mathrm{~mm}$. The horizontal dashed line is the Rayleigh wave speed of the material. The grey areas are the x-locations of the inclusions.

\section{Conclusions}

We have numerically analyzed the pre-strained plate configuration to study the influence of distant material heterogeneities on dynamic failure. PMMA is used as the plate material. With this setup, the effects of elastic field resulting from pre-straining and stress wave interactions throughout the crack propagation are isolated by defining stiffer and denser media in the plate. The crack tip is repelled by the stiffer inclusions, while it is attracted to the identical-shaped denser ones.

Lower steady-state crack velocities are obtained when the PMMA strip is narrowed down by defining denser media at the top and bottom of the plate. We have shown that a portion of the total stored elastic energy can be trapped in the dense regions, thereby limiting the energy flow to the crack tip. The portion that is still effective in the process of crack propagation is calculated by multiplying the total stored energy by the volume fraction of actual PMMA 

crack velocities at different applied displacements collapse on the same curve. We observed crack branching at much lower velocities than the limiting velocity of our system, providing further evidence that the crack velocity is not a valid criterion for the branching phenomena.

260 geneities. There are engineering materials such as metals, ceramics, polymers and elastomers, the combinations of which can give the discussed stiffness and density ratios by various designs, see an Ashby plot of Young's modulus and density, for example [41]. However, the recommendations for possible material combinations are outside the scope of this work as this requires a thorough analysis of considered materials, design and production phases.

\section{References}

[1] J. Bleyer, C. Roux-Langlois, J.-F. Molinari, Dynamic crack propagation with a variational phase-field model: limiting speed, crack branching and velocity-toughening mechanisms, International Journal of Fracture (2016) $1-22$.

[2] K. Ravi-Chandar, Dynamic fracture, Elsevier, 2004.

[3] A. Sagy, G. Cohen, Z. Reches, J. Fineberg, Dynamic fracture of granular material under quasi-static loading, Journal of Geophysical Research: Solid Earth 111 (2006).

[4] G. Albertini, D. Kammer, Supershear transition of dynamic mode II fracture in heterogeneous elastic media, Journal of Mechanics and Physics of Solids (2017). Submitted.

[5] D. Fisher, K. Dahmen, S. Ramanathan, Y. Ben-Zion, Statistics of earth280 quakes in simple models of heterogeneous faults, Physical Review Letters 78 (1997) 4885. 
[6] Y. Ben-Zion, Collective behavior of earthquakes and faults: Continuumdiscrete transitions, progressive evolutionary changes, and different dynamic regimes, Reviews of Geophysics 46 (2008).

[7] Z. Zhang, F. Wu, W. Gao, J. Tan, Z. Wang, M. Stoica, J. Das, J. Eckert, B. Shen, A. Inoue, Wavy cleavage fracture of bulk metallic glass, Applied Physics Letters 89 (2006) 251917.

[8] P. Murali, T. Guo, Y. Zhang, R. Narasimhan, Y. Li, H. Gao, Atomic scale fluctuations govern brittle fracture and cavitation behavior in metallic glasses, Physical Review Letters 107 (2011) 215501.

[9] F. Zhou, Study on the macroscopic behavior and the microscopic process of dynamic crack propagation, Ph.D. thesis, PhD dissertation. The University of Tokyo, Tokyo, 1996.

[10] F. Zhou, J.-F. Molinari, T. Shioya, A rate-dependent cohesive model for simulating dynamic crack propagation in brittle materials, Engineering Fracture Mechanics 72 (2005) 1383-1410.

[11] A. Livne, G. Cohen, J. Fineberg, Universality and hysteretic dynamics in rapid fracture, Physical Review Letters 94 (2005) 224301.

[12] T. Goldman, A. Livne, J. Fineberg, Acquisition of inertia by a moving crack, Physical Review Letters 104 (2010) 114301.

[13] C. Wolff, N. Richart, J.-F. Molinari, A non-local continuum damage approach to model dynamic crack branching, International Journal for $\mathrm{Nu}-$ merical Methods in Engineering 101 (2015) 933-949.

[14] D. Dugdale, Yielding of steel sheets containing slits, Journal of the Mechanics and Physics of Solids 8 (1960) 100-104.

[15] G. Barenblatt, The mathematical theory of equilibrium cracks in brittle fracture, Advances in Applied Mechanics 7 (1962) 55-129. 
[16] G. Camacho, M. Ortiz, Computational modelling of impact damage in brittle materials, International Journal of Solids and Structures 33 (1996) 2899-2938.

[17] M. Ortiz, A. Pandolfi, Finite-deformation irreversible cohesive elements for three-dimensional crack-propagation analysis, International Journal for Numerical Methods in Engineering 44 (1999) 1267-1282.

[18] W. Chen, G. Ravichandran, Static and dynamic compressive behavior of aluminum nitride under moderate confinement, Journal of the American Ceramic Society 79 (1996) 579-584.

[19] G. Ruiz, M. Ortiz, A. Pandolfi, Three-dimensional finite-element simulation of the dynamic brazilian tests on concrete cylinders, International Journal for Numerical Methods in Engineering 48 (2000) 963-994.

320 [20] A. Livne, E. Bouchbinder, J. Fineberg, Breakdown of linear elastic fracture mechanics near the tip of a rapid crack, Physical Review Letters 101 (2008) 264301.

[21] A. Livne, E. Bouchbinder, I. Svetlizky, J. Fineberg, The near-tip fields of fast cracks, Science 327 (2010) 1359-1363.

325 [22] M. Ramulu, A. Kobayashi, Mechanics of crack curving and branching - a dynamic fracture analysis, in: Dynamic fracture, Springer, 1985, pp. 61-75.

[23] J. Fineberg, S. Gross, M. Marder, H. Swinney, Instability in dynamic fracture, Physical Review Letters 67 (1991) 457.

[24] E. Sharon, S. Gross, J. Fineberg, Local crack branching as a mechanism for instability in dynamic fracture, Physical Review Letters 74 (1995) 5096.

[25] E. Sharon, J. Fineberg, Confirming the continuum theory of dynamic brittle fracture for fast cracks, Nature 397 (1999) 333-335. 
[26] X.-P. Xu, A. Needleman, Numerical simulations of fast crack growth in brittle solids, Journal of the Mechanics and Physics of Solids 42 (1994) $1397-1434$.

[27] J.-H. Song, H. Wang, T. Belytschko, A comparative study on finite element methods for dynamic fracture, Computational Mechanics 42 (2008) 239250.

[28] Y. Ha, F. Bobaru, Studies of dynamic crack propagation and crack branching with peridynamics, International Journal of Fracture 162 (2010) 229244.

[29] T. Belytschko, W. K. Liu, B. Moran, K. Elkhodary, Nonlinear finite elements for continua and structures, 2 ed., John Wiley \& Sons, 2014.

[30] R. Courant, K. Friedrichs, H. Lewy, On the partial difference equations of mathematical physics, IBM Journal of Research and Development 11 (1967) 215-234.

[31] A. Palmer, J. Rice, The growth of slip surfaces in the progressive failure of over-consolidated clay, Proceedings of the Royal Society of London. A. Mathematical and Physical Sciences 332 (1973) 527-548.

[32] A. Hillerborg, M. Modeer, P. Petersson, Analysis of crack formation and crack growth in concrete by means of fracture mechanics and finite elements, Cement and Concrete Research 6 (1976) 773-782.

[33] L. Snozzi, A. Caballero, J. Molinari, Influence of the meso-structure in dynamic failure simulation of concrete under tensile loading, Cement and Concrete Research 41 (2011) 1130-1142.

[34] J. Fineberg, S. Gross, M. Marder, H. Swinney, Instability in the propagation of fast cracks, Physical Review B 45 (1992) 5146.

[35] J.-F. Molinari, G. Gazonas, R. Raghupathy, A. Rusinek, F. Zhou, The cohesive element approach to dynamic fragmentation: the question of energy 
convergence, International Journal for Numerical Methods in Engineering 69 (2007) 484-503.

[36] M. Marder, New dynamical equation for cracks, Physical Review Letters 66 (1991) 2484.

[37] M. Marder, Adiabatic equation for cracks, Philosophical Magazine B 78 (1998) 203-214.

[38] E. Yoffe, The moving griffith crack, The London, Edinburgh, and Dublin Philosophical Magazine and Journal of Science 42 (1951) 739-750.

[39] T. Seelig, D. Gross, On the interaction and branching of fast running cracks - a numerical investigation, Journal of the Mechanics and Physics of Solids 47 (1999) 935-952.

[40] K. Ravi-Chandar, W. Knauss, An experimental investigation into dynamic fracture: III. on steady-state crack propagation and crack branching, International Journal of Fracture 26 (1984) 141-154.

[41] N. Fleck, V. Deshpande, M. Ashby, Micro-architectured materials: past, present and future, in: Proceedings of the Royal Society of London A: Mathematical, Physical and Engineering Sciences, volume 466, The Royal Society, 2010, pp. 2495-2516. 